\title{
Molecular Mechanisms Underlying Accelerated Aging by Defects in the FGF23-Klotho System
}

\author{
Makoto Kuro-o $\mathbb{D i D}^{1,2}$ \\ ${ }^{1}$ Division of Anti-Aging Medicine, Center for Molecular Medicine, Jichi Medical University, 3311-1 Yakushiji, Shimotsuke, \\ Tochigi 329-0498, Japan \\ ${ }^{2}$ Department of Internal Medicine, Division of Mineral Metabolism, University of Texas Southwestern Medical Center, \\ 5323 Harry Hines Blvd., Dallas, TX 75390-9072, USA \\ Correspondence should be addressed to Makoto Kuro-o; mkuroo@jichi.ac.jp
}

Received 14 February 2018; Accepted 12 April 2018; Published 21 May 2018

Academic Editor: Nicolas Verheyen

Copyright ( $\odot 2018$ Makoto Kuro-o. This is an open access article distributed under the Creative Commons Attribution License, which permits unrestricted use, distribution, and reproduction in any medium, provided the original work is properly cited.

\begin{abstract}
The basic research of aging has been primarily focused on elucidating mechanisms of aging and longevity that are evolutionarily conserved from yeasts to primates. Such efforts have culminated in the notion that (1) senescence at the cellular level is associated with aging at the organismal level and that (2) calorie restriction and growth suppression decelerate aging. However, these important findings in the basic research have not necessarily been linked to improvement of daily medical practice in the aging society. It has become increasingly important to investigate mechanisms of aging unique to mammals or humans and apply the research fruits for the treatment of major age-related disorders to extend the health span. Seminal studies on the klotho mouse, a mutant exhibiting a premature aging syndrome, have identified phosphate as a proaging factor in mammals. In this review, mechanisms of phosphateinduced premature aging and potential therapeutic targets will be discussed, which may be directly applicable for developing novel strategies for the treatment of chronic kidney disease and its complications.
\end{abstract}

\section{Introduction}

In 1997, a mutant mouse that displayed phenotypes resembling human premature aging syndromes was reported [1]. The aging-like phenotypes included shortened lifespan, atrophy of multiple organs (gonads, thymus, skin, etc.), vascular calcification, cardiac hypertrophy, osteopenia, sarcopenia, cognition impairment, hearing disturbance, and frailty. These symptoms were attributed to abolished expression of the klotho gene caused by an insertional mutation at its $5^{\prime}$ flanking region [1] associated with DNA hypermethylation [2]. The gene was named after a Greek goddess Klotho who spins the thread of life. The klotho gene encodes a single-pass transmembrane protein and is expressed only in a few tissues such as renal tubular cells [1] and parathyroid chief cells [3]. It was a new gene at that time and different from any genes defective in human premature aging syndromes that encode cytoplasmic or nuclear proteins involved in DNA repair.

In 2006, Klotho protein function was identified as a receptor for fibroblast growth factor-23 (FGF23) [4]. This discovery was prompted by the fact that $\mathrm{Fgf2} 23^{-/-}$mice [5] and Klotho-deficient $(k l / k l)$ mice exhibited identical phenotypes, namely, aging-like phenotypes associated with disturbed mineral metabolism. FGF23 is a peptide hormone secreted from osteocytes and/or osteoblasts upon phosphate intake. FGF23 then acts on the kidney to increase urinary phosphate excretion by suppressing phosphate reabsorption in renal proximal tubules, thereby maintaining the phosphate balance. FGF23 also suppresses synthesis of the active form of vitamin $\mathrm{D}\left(1,25\right.$-dihydroxyvitamin $\left.\mathrm{D}_{3}\right)$ in proximal tubules. Unlike the other members of the FGF family, FGF23 requires Klotho for binding and activating its cognate fibroblast growth factor receptors (FGFRs) [6].

Recently, the crystal structure of the Klotho-FGFR1cFGF23 complex was resolved [7]. The extracellular domain of Klotho protein has a long arm that directly interacts with FGFR1c, termed the receptor-binding arm (RBA). By analogy from the protein structure of another member of the Klotho family ( $\beta$ Klotho) [8], the RBA of Klotho is supposed to have an intrinsically disordered structure and 
adopts a fixed configuration after binding to the extracellular domain of FGFR1c [9]. FGF23 fits into the groove generated between Klotho and FGFR1c. These findings have provided a structural basis for the unique feature that FGF23 requires Klotho to exert its hormonal actions.

\section{Evolution of the Klotho Gene}

During the evolution of life on earth, the first organisms that stored phosphorus in their body are bony fishes (Osteichthyes) that appeared in the Devonian period ( 400 million years ago) $[6,10]$. They accumulated phosphorus in the bone as calcium phosphate $(\mathrm{CaPi})$. At that time, some other organisms such as sharks (Chondrichthyes) and shellfish (Mollusca) had skeletons made of cartilage and calcium carbonate. When compared with these skeletons, the bone made of $\mathrm{CaPi}$ or hydroxyapatite was much harder and stronger. It might be a prerequisite for the evolution of terrestrial animals to acquire hard and strong skeletons made of $\mathrm{CaPi}$, which enable them to support their own body weight in the absence of buoyancy. In this context, it is interesting to note that orthologs of the klotho gene exist only in vertebrates harboring the bone made of $\mathrm{CaPi}$. The klotho gene might have been evolved under the necessity in the regulation of phosphate metabolism.

\section{Phosphate and Aging}

The fact that FGF23 functions as a phosphaturic hormone as well as a counterregulatory hormone against vitamin D in a Klotho-dependent manner implies that the fundamental pathophysiology of mice lacking either FGF23 or Klotho is phosphate retention and vitamin D intoxication. In fact, these mice suffer from hyperphosphatemia and hypervitaminosis $\mathrm{D}$ [11]. Provided that the disturbed mineral metabolism is responsible for the aging-like phenotypes, these mice should be rescued by resolving phosphate retention and/or vitamin $\mathrm{D}$ intoxication.

Several laboratories tested this notion and identified interventions that rescue FGF23-deficient mice and/or Klotho-deficient mice from the aging-like symptoms [12]. Such interventions included feeding of vitamin D-deficient diet or low phosphate diet, ablation of the vitamin $\mathrm{D}$ receptor (Vdr) gene, the Cyp27b1 gene, and the sodium-dependent phosphate transporter type IIa (Npt2a) gene (Table 1). The Cyp27b1 gene encodes $1 \alpha$-hydroxylase, the enzyme necessary for synthesis of 1,25-dihydroxyvitamin $\mathrm{D}_{3}$. The Npt2a gene is expressed in renal proximal tubules, where Npt2a protein is located on the apical brush border membrane and functions as the major entry gate of phosphate reabsorption from the tubular fluid in the process of transcellular phosphate transport. Among these interventions, vitamin D-deficient diet and ablation of the $V d r$ or Cyp27b1 gene lowered both serum phosphate and 1,25-dihydroxyvitamin $\mathrm{D}_{3}$ levels. In contrast, low phosphate diet and ablation of the Npt2a gene lowered serum phosphate levels but increased 1,25-dihydroxyvitamin $\mathrm{D}_{3}$ levels, which is regarded as a compensatory attempt to maximize phosphate absorption from the diet. Despite the further increase in 1,25-dihydroxyvitamin $D_{3}$, these
TABLE 1: Interventions that alleviate the aging-like phenotypes in Klotho-deficient mice and/or FGF23-deficient mice and associated changes in serum levels of phosphate and 1,25-dihydroxyvitamin $\mathrm{D}_{3}$ (Vitamin D).

\begin{tabular}{lcc}
\hline \multirow{2}{*}{ Interventions } & \multicolumn{2}{c}{ Changes in serum levels } \\
& Phosphate & Vitamin D \\
\hline Vitamin D-deficient diet & $\downarrow$ & $\downarrow$ \\
Ablation of vitamin D receptor & $\downarrow$ & $\downarrow$ \\
Ablation of $1 \alpha$-hydroxylase & $\downarrow$ & $\downarrow$ \\
Ablation of Npt2a & $\downarrow$ & $\uparrow$ \\
Low phosphate diet & $\downarrow$ & $\uparrow$ \\
\hline
\end{tabular}

interventions alleviated the aging-like phenotypes, indicating that it was not vitamin $\mathrm{D}$ but phosphate that was responsible for the premature aging syndrome. These observations have led us to conclude that phosphate accelerates aging [13]. Of note, association between accelerated aging and elevated serum phosphate levels is also observed in some patients with Hutchinson-Gilford syndrome, a rare hereditary progeroid disease caused by mutation in the lamin A gene, although high phosphate may not be the primary reason for their premature aging symptoms [14].

\section{Phosphate Toxicity}

It has been known in tissue culture experiments that extracellular phosphate is toxic to various types of cells at high concentration. For example, increase in the phosphate concentration in the tissue culture medium was reported to induce cell damage, apoptosis, and calcification in vascular endothelial cells and smooth muscle cells $[15,16]$. The "phosphate toxicity" may contribute to the mechanism by which phosphate accelerates aging.

The phosphate toxicity observed in cultured cells was alleviated by adding phosphonoformic acid (PFA) to the high phosphate medium [16], which inhibits sodium-dependent phosphate cotransporter (Npt). Thus, it was thought necessary for phosphate to enter the cell to exert the cytotoxic effects. However, several lines of evidence have argued against this notion. First, the ability of PFA to inhibit phosphateinduced calcification of cultured vascular smooth muscle cells was shown independent of cellular phosphate uptake. Vascular smooth muscle cells express type III Npt involved in house-keeping cellular phosphate uptake, but not type II Npt involved in transepithelial phosphate resorption in renal proximal tubular cells. PFA inhibits type II Npt but barely inhibits type III Npt [17]. Instead, PFA inhibits formation of $\mathrm{CaPi}$ crystals like pyrophosphate and bisphosphonate, suggesting that the ability of PFA to inhibit calcification may be actually attributed to its ability to inhibit $\mathrm{CaPi}$ crystal formation [18]. Second, addition of insoluble CaPi crystals to the medium was shown to induce cellular damage and calcification $[19,20]$. The concentration of calcium and phosphate ions in regular tissue culture medium is around $2 \mathrm{mM}$ and $1 \mathrm{mM}$, respectively. Because this is a supersaturated condition regarding various types of $\mathrm{CaPi}$ precipitates, a small increase in the phosphate concentration can induce 
precipitation of $\mathrm{CaPi}$. Therefore, the true culprit of phosphate toxicity may not be phosphate per se but CaPi crystals. Unfortunately, this possibility has not been explored in depth.

\section{Calciprotein Particles (CPP)}

Acquisition of the bone made of $\mathrm{CaPi}$ requires a defense mechanism that prevents $\mathrm{CaPi}$ from growing into large crystals in the extraosseous tissues and in the extracellular fluid. The extracellular fluid in mammals is supersaturated in terms of calcium and phosphate ions and therefore at high risk for $\mathrm{CaPi}$ precipitation. However, $\mathrm{CaPi}$ crystals never grow in the blood, because serum protein fetuin-A absorbs tiny $\mathrm{CaPi}$ precipitates and prevents them from growing into large crystals. As a result, aggregates of CaPi-laden fetuinA molecules are generated and dispersed in the blood as colloidal particles, which are called calciprotein particles (CPP) [21]. Thus, CaPi in the blood exist in the form of CPP.

Although less potent than $\mathrm{CaPi}, \mathrm{CPP}$ can also induce calcification in vascular smooth muscle cells [22] and innate immune responses in macrophages in culture [23]. Recent clinical studies have indicated that serum CPP levels are increased with decline of renal function and associated with clinical parameters for vascular stiffness, vascular calcification, and noninfectious chronic inflammation [24,25]. These observations have raised the possibility that the relation between CPP and these pathological conditions may not merely be an association but a causation. Namely, the ability of CPP to behave like a "pathogen" may contribute to arteriosclerosis and chronic inflammation in patients with chronic kidney disease (CKD), a clinical model for accelerated aging [26].

Formation of $\mathrm{CPP}$ is primarily a physicochemical process affected not only by concentration of calcium and phosphate ions but also by various factors such as $\mathrm{pH}$, temperature, ionic strength, incubation time, composition, and concentration of other ions and proteins in the solution. Depending on these parameters, physical properties of CPP, including particle size distribution, protein-mineral ratio, and the surface charge (zeta potential), and the $\mathrm{CaPi}$ phase (amorphous or crystalline) should vary. In fact, when formation of CPP was induced in test tubes by adding calcium $(10 \mathrm{mM})$ and phosphate $(6 \mathrm{mM})$ to serum, physical properties of CPP changed dynamically over time [27]. Spheroidal CPP carrying amorphous $\mathrm{CaPi}$ were generated within an hour, which were smaller than $100 \mathrm{~nm}$ in diameter and called primary CPP. Thereafter, a sudden increase in turbidity was observed due to the Tyndall phenomenon caused by formation of ellipsoidal CPP carrying crystalline $\mathrm{CaPi}$, which were larger than primary CPP and called secondary CPP. Transition from primary CPP to secondary CPP occurred spontaneously within a few hours after addition of calcium and phosphate. The time required for formation of secondary CPP serves as a marker for the serum propensity for CPP formation. The shorter the time, the higher the serum propensity for CPP formation. A clinical study using stage 3 and 4 CKD patients showed that the serum propensity for CPP formation was associated with vascular stiffness and all-cause mortality $[25,28]$. Thus, this assay is thought useful not only as a clinical test for evaluation of the risk for arteriosclerosis but also as a support of the notion that CPP may contribute to acceleration of CKD complications and perhaps aging.

\section{CPP Levels in the Blood}

Several different methods have been reported for quantification of CPP levels in the blood. Based on the assumption that CPP are precipitated by centrifugation at $16,000 \sim 30,000 \mathrm{~g}$ for 120 minutes, difference in the serum fetuin-A levels between before and after the centrifugation has been used as a surrogate of serum CPP levels $[24,25]$. Thereafter, novel CPP assays were reported that directly detected CPP in the serum/plasma using a fluorescent probe for $\mathrm{CaPi}$ crystals $[29,30]$. Of note, one of these studies identified lowdensity CPP that were not precipitated by the centrifugation and demonstrated that serum phosphate and age were the two major independent predictor variables of plasma CPP levels in the population without hyperphosphatemia [30]. Because serum phosphate levels, even within the normal range, were reported to correlate with all-cause mortality [31], it is intriguing to speculate that high blood phosphate is a risk for high blood CPP that may accelerate aging and increase all-cause mortality through inducing chronic noninfectious inflammation. In support of this speculation, serum phosphate levels inversely correlate with longevity in mammals [12].

\section{CPP in the Urine}

Renal toxicity of dietary phosphate load was reported several decades ago [32]. In that report, normal rats and uninephrectomized rats were placed on diet containing various amount of phosphate for 18 weeks and then evaluated for renal histology. The conclusion was that phosphate excretion per nephron, but not serum phosphate, was correlated with a score of histological changes that reflected severity of tubular damage and interstitial fibrosis. Although the FGF23-Klotho endocrine system was not known at that time, if serum FGF23 had been measured, it should have been positively correlated with phosphate excretion per nephron. Because FGF23 suppresses phosphate resorption at the proximal tubules, FGF23 should increase phosphate concentration and thus the risk for formation of $\mathrm{CaPi}$ in the proximal tubular fluid. Indeed, micropuncture studies indicated that concentration of calcium and phosphate ions in the proximal tubular fluid could exceed the solubility limit upon dietary phosphate load [33]. Although it remains to be determined whether CPP indeed appear in the tubular lumen, it is possible that high FGF23 that increases phosphate excretion per nephron may induce tubular damage and renal fibrosis by promoting CPP formation in the tubular fluid, regardless of serum phosphate levels.

\section{Phosphate Restriction in Future}

Since hyperphosphatemia was identified as a risk for vascular calcification and cardiovascular events, phosphate restriction by means of diet counseling and administration of phosphate 
TABLE 2: Comparison between the current paradigm and proposed paradigm for phosphate restriction.

\begin{tabular}{lcc}
\hline & \multicolumn{1}{c}{ Current paradigm } & Proposed paradigm \\
\hline Rationale & Hyperphosphatemia is a risk for vascular calcification and \\
mortality & To lower serum phosphate levels & $\begin{array}{c}\text { Increase in phosphate excretion per nephron is a risk for renal } \\
\text { tubular damage }\end{array}$ \\
Goal & Vascular calcification, cardiovascular events, death & To lower serum FGF23 levels \\
Outcome & Renal tubular damage \\
Indication & Hyperphosphatemia $(\sim 0.35 \%$ of the total CKD patients $)$ & Hyper-FGF23-emia ( $\sim 8 \%$ of the total CKD patients $)$ \\
\hline
\end{tabular}

binders has been justified for CKD patients with hyperphosphatemia [34-36]. The goal of phosphate restriction is to reduce serum phosphate levels. However, it should be noted that hyperphosphatemia is a terminal symptom during CKD progression observed in patients with end-stage renal disease (ESRD), whose residual nephron number is estimated as $1 / 10$ or less of normal individuals [37]. In fact, phosphate binders are prescribed mostly for dialysis patients. Although phosphate binders are expected to improve prognosis of CKD patients, a recent network meta-analysis study failed to show evidence that phosphate binder treatment reduced mortality compared with placebo in ESRD patients [38]. It may be necessary to reevaluate the current paradigm for phosphate restriction.

Because increase in phosphate excretion per nephron can induce tubular and interstitial damage, it is reasonable to start phosphate restriction when phosphate excretion per nephron is increased. Because FGF23 is a phosphaturic hormone that increases phosphate excretion per nephron, FGF23 likely serves as a surrogate marker for phosphate excretion per nephron. Thus, increase in FGF23 should be recognized as a sign of excess phosphate intake relative to the residual nephron number and a ground for starting phosphate restriction regardless of serum phosphate levels. Because FGF23 increases long before hyperphosphatemia ensues during CKD progression [39], phosphate binders should be started at stages 2 or 3 when serum FGF23 levels, but not serum phosphate levels, start increasing. In this new paradigm for phosphate restriction, the goal is to lower serum FGF23 levels, but not serum phosphate levels. The outcome to be evaluated must be tubular damage, but not vascular calcification, cardiovascular events, or mortality (Table 2). This new paradigm requires justification by clinical studies.

\section{Conclusion}

Discovery of the klotho gene and its function as the obligate coreceptor for FGF23 revealed a novel endocrine axis indispensable for maintaining phosphate homeostasis. The fact that Klotho- or FGF23-deficient mice exhibit premature aging and are restored from the aging-like phenotypes by dietary phosphate restriction leads to the notion that phosphate accelerates aging. Phosphate exerts its toxicity when it binds to calcium and becomes $\mathrm{CaPi}$. In the blood, $\mathrm{CaPi}$ binds to serum protein fetuin-A and forms colloidal particles termed CPP. CPP increase with age and serum phosphate levels. CPP can induce vascular endothelial damage, smooth muscle calcification, and innate immune responses, leading to arteriosclerosis and chronic noninfectious inflammation.
In the renal tubular fluid, CPP may appear when phosphate excretion per nephron is increased by FGF23. CPP induce renal tubular damage and interstitial fibrosis to accelerate kidney aging and exacerbate CKD. Provided that CPP, but not phosphate per se, are responsible for phosphate toxicity and thus accelerate aging, CPP may be justified as a novel therapeutic target for aging and age-related disorders.

\author{
Abbreviations \\ FGF23: Fibroblast growth factor-23 \\ FGFR: Fibroblast growth factor receptor \\ Npt2a: Sodium-dependent phosphate transporter \\ type IIa \\ Npt3: Sodium-dependent phosphate transporter \\ type III \\ PFA: Phosphonoformic acid \\ Vdr: Vitamin D receptor \\ Cyp27b1: Cytochrome P450 family 27 subfamily B \\ member 1 \\ CaPi: Calcium phosphate \\ CPP: Calciprotein particles \\ CKD: Chronic kidney disease.
}

\section{Conflicts of Interest}

The author declares no conflicts of interest regarding the publication of this paper.

\section{Acknowledgments}

This article is supported in part by Japan Society for the Promotion of Science (Grant nos. 16H05302, 16K15470) and AMED-CREST, Japan Agency for Medical Research and Development.

\section{References}

[1] M. Kuro-o, Y. Matsumura, H. Aizawa et al., "Mutation of the mouse klotho gene leads to a syndrome resembling ageing," Nature, vol. 390, no. 6655, pp. 45-51, 1997.

[2] M. Azuma, D. Koyama, J. Kikuchi et al., "Promoter methylation confers kidney-specific expression of the Klotho gene," The FASEB Journal, vol. 26, no. 10, pp. 4264-4274, 2012.

[3] I. Z. Ben-Dov, H. Galitzer, V. Lavi-Moshayoff et al., "The parathyroid is a target organ for FGF23 in rats," The Journal of Clinical Investigation, vol. 117, no. 12, pp. 4003-4008, 2007.

[4] H. Kurosu, Y. Ogawa, M. Miyoshi et al., "Regulation of fibroblast growth factor-23 signaling by Klotho," The Journal of Biological Chemistry, vol. 281, no. 10, pp. 6120-6123, 2006. 
[5] T. Shimada, M. Kakitani, and Y. Yamazaki, "Targeted ablation of Fgf23 demonstrates an essential physiological role of FGF23 in phosphate and vitamin D metabolism," The Journal of Clinical Investigation, vol. 113, no. 4, pp. 561-568, 2004.

[6] M. C. Hu, K. Shiizaki, M. Kuro-O, and O. W. Moe, "Fibroblast growth factor 23 and klotho: physiology and pathophysiology of an endocrine network of mineral metabolism," Annual Review of Physiology, vol. 75, pp. 503-533, 2013.

[7] G. Chen, Y. Liu, R. Goetz et al., " $\alpha$-Klotho is a non-enzymatic molecular scaffold for FGF23 hormone signalling," Nature, vol. 553, no. 7689, pp. 461-466, 2018.

[8] S. Lee, J. Choi, J. Mohanty et al., "Structures of $\beta$-klotho reveal a 'zip code'-like mechanism for endocrine FGF signalling," Nature, vol. 553, no. 7689, pp. 501-505, 2018.

[9] M. Kuro-o, "Ageing-related receptors resolved," Nature, vol. 553, no. 7689, pp. 409-410, 2018.

[10] P. Stenvinkel, J. Painer, M. Kuro-o et al., "Novel treatment strategies for chronic kidney disease: insights from the animal kingdom," Nature Reviews Nephrology, vol. 14, no. 4, pp. 265284, 2018.

[11] H. Tsujikawa, Y. Kurotaki, T. Fujimori, K. Fukuda, and Y.-I. Nabeshima, "Klotho, a gene related to a syndrome resembling human premature aging, functions in a negative regulatory circuit of vitamin D endocrine system," Molecular Endocrinology, vol. 17, no. 12, pp. 2393-2403, 2003.

[12] M. Kuro-o, "A potential link between phosphate and agingLessons from Klotho-deficient mice," Mechanisms of Ageing and Development, vol. 131, no. 4, pp. 270-275, 2010.

[13] M. Kuro-o, "Klotho, phosphate and FGF-23 in ageing and disturbed mineral metabolism," Nature Reviews Nephrology, vol. 9, no. 11, pp. 650-660, 2013.

[14] M. A. Merideth, L. B. Gordon, S. Clauss et al., "Phenotype and course of Hutchinson-Gilford progeria syndrome," The New England Journal of Medicine, vol. 358, no. 6, pp. 592-604, 2008.

[15] G. S. Di Marco, M. Hausberg, U. Hillebrand et al., "Increased inorganic phosphate induces human endothelial cell apoptosis in vitro," American Journal of Physiology-Renal Physiology, vol. 294, no. 6, pp. F1381-F1387, 2008.

[16] S. Jono, M. D. McKee, C. E. Murry et al., "Phosphate regulation of vascular smooth muscle cell calcification," Circulation Research, vol. 87, no. 7, pp. e10-e17, 2000.

[17] R. Villa-Bellosta, Y. E. Bogaert, M. Levi, and V. Sorribas, "Characterization of phosphate transport in rat vascular smooth muscle cells: implications for vascular calcification," Arteriosclerosis, Thrombosis, and Vascular Biology, vol. 27, no. 5, pp. 1030-1036, 2007.

[18] R. Villa-Bellosta and V. Sorribas, "Phosphonoformic acid prevents vascular smooth muscle cell calcification by inhibiting calcium-phosphate deposition," Arteriosclerosis, Thrombosis, and Vascular Biology, vol. 29, no. 5, pp. 761-766, 2009.

[19] A. E. Ewence, M. Bootman, H. L. Roderick et al., "Calcium phosphate crystals induce cell death in human vascular smooth muscle cells: a potential mechanism in atherosclerotic plaque destabilization," Circulation Research, vol. 103, no. 5, pp. e28e34, 2008

[20] A. P. Sage, J. Lu, Y. Tintut, and L. L. Demer, "Hyperphosphatemia-induced nanocrystals upregulate the expression of bone morphogenetic protein-2 and osteopontin genes in mouse smooth muscle cells in vitro," Kidney International, vol. 79, no. 4, pp. 414-422, 2011.
[21] A. Heiss, V. Pipich, W. Jahnen-Dechent, and D. Schwahn, "Fetuin-A is a mineral carrier protein: Small angle neutron scattering provides new insight on fetuin-A controlled calcification inhibition," Biophysical Journal, vol. 99, no. 12, pp. 3986-3995, 2010.

[22] P. Aghagolzadeh, M. Bachtler, R. Bijarnia et al., "Calcification of vascular smooth muscle cells is induced by secondary calciprotein particles and enhanced by tumor necrosis factor-

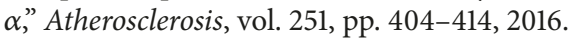

[23] E. R. Smith, E. Hanssen, L. P. McMahon, and S. G. Holt, "FetuinA-containing calciprotein particles reduce mineral stress in the macrophage," PLoS ONE, vol. 8, no. 4, Article ID e60904, 2013.

[24] T. Hamano, I. Matsui, S. Mikami et al., "Fetuin-mineral complex reflects extraosseous calcification stress in CKD," Journal of the American Society of Nephrology, vol. 21, pp. 1998-2007, 2010.

[25] E. R. Smith, M. L. Ford, L. A. Tomlinson, C. Rajkumar, L. P. McMahon, and S. G. Holt, "Phosphorylated fetuin-Acontaining calciprotein particles are associated with aortic stiffness and a procalcific milieu in patients with pre-dialysis CKD," Nephrology Dialysis Transplantation, vol. 27, no. 5, pp. 1957-1966, 2012.

[26] P. Stenvinkel and T. E. Larsson, "Chronic kidney disease: A clinical model of premature aging," American Journal of Kidney Diseases, vol. 62, no. 2, pp. 339-351, 2013.

[27] A. Pasch, S. Farese, S. Gräber et al., "Nanoparticle-based test measures overall propensity for calcification in serum," Journal of the American Society of Nephrology, vol. 23, no. 10, pp. 17441752, 2012.

[28] E. R. Smith, M. L. Ford, L. A. Tomlinson et al., "Serum calcification propensity predicts all-cause mortality in predialysis CKD," Journal of the American Society of Nephrology, vol. 25, no. 2, pp. 339-348, 2014.

[29] E. R. Smith, T. D. Hewitson, M. M. X. Cai et al., "A novel fluorescent probe-based flow cytometric assay for mineralcontaining nanoparticles in serum," Scientific Reports, vol. 7, no. 1, article no. 5686, 2017.

[30] Y. Miura, Y. Iwazu, K. Shiizaki et al., "Identification and quantification of plasma calciprotein particles with distinct physical properties in patients with chronic kidney disease," Scientific Reports, vol. 8, no. 1, 2018.

[31] M. Tonelli, F. Sacks, M. Pfeffer, Z. Gao, and G. Curhan, "Relation between serum phosphate level and cardiovascular event rate in people with coronary disease," Circulation, vol. 112, no. 17, pp. 2627-2633, 2005.

[32] L. L. Haut, A. C. Alfrey, S. Guggenheim, B. Buddington, and N. Schrier, "Renal toxicity of phosphate in rats," Kidney International, vol. 17, no. 6, pp. 722-731, 1980.

[33] K. Lau, "Phosphate excess and progressive renal failure: The precipitation-calcification hypothesis," Kidney International, vol. 36, no. 5, pp. 918-937, 1989.

[34] B. Kestenbaum, J. N. Sampson, K. D. Rudser et al., "Serum phosphate levels and mortality risk among people with chronic kidney disease," Journal of the American Society of Nephrology, vol. 16, no. 2, pp. 520-528, 2005.

[35] S. K. Ganesh, A. G. Stack, N. W. Levin, T. Hulbert-Shearon, and F. K. Port, "Association of elevated serum $\mathrm{PO}(4), \mathrm{Ca} \times \mathrm{PO}(4)$ product, and parathyroid hormone with cardiac mortality risk in chronic hemodialysis patients," Journal of the American Society of Nephrology, vol. 12, no. 10, pp. 2131-2138, 2001.

[36] K. J. Martin and E. A. González, "Prevention and control of phosphate retention/ hyperphosphatemia in CKD-MBD: what 
is normal, when to start, and how to treat?" Clinical Journal of the American Society of Nephrology, vol. 6, no. 2, pp. 440-446, 2011.

[37] E. Slatopolsky, "The intact nephron hypothesis: the concept and its implications for phosphate management in CKD-related mineral and bone disorder," Kidney International Supplements, no. 121, pp. S3-8, 2011.

[38] S. C. Palmer, S. Gardner, M. Tonelli et al., "Phosphate-Binding Agents in Adults With CKD: A Network Meta-analysis of Randomized Trials," American Journal of Kidney Diseases, vol. 68, no. 5, pp. 691-702, 2016.

[39] T. Isakova, P. Wahl, G. S. Vargas et al., "Fibroblast growth factor 23 is elevated before parathyroid hormone and phosphate in chronic kidney disease," Kidney International, vol. 79, no. 12, pp. 1370-1378, 2011. 


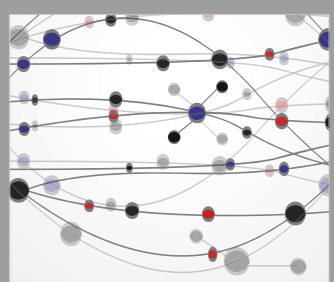

The Scientific World Journal
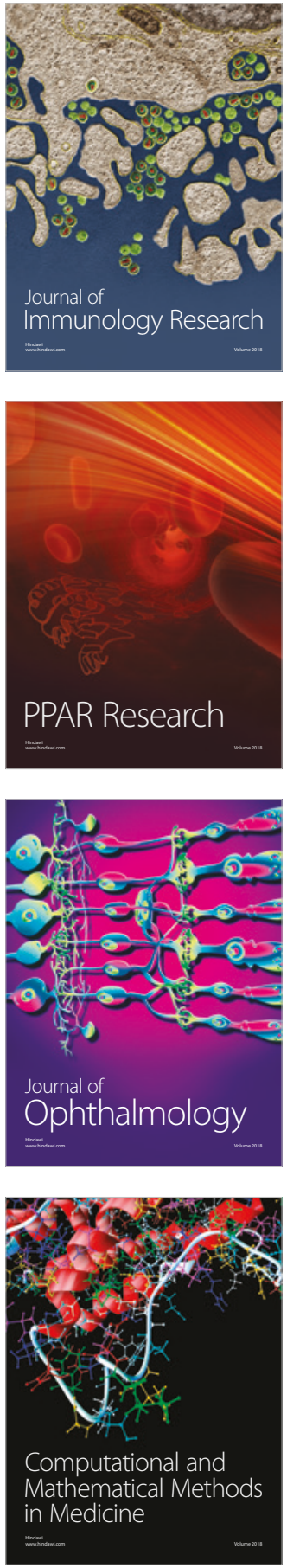

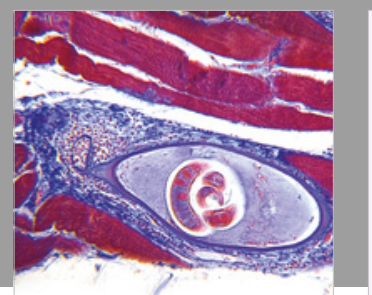

Gastroenterology Research and Practice

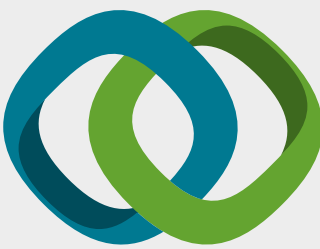

\section{Hindawi}

Submit your manuscripts at

www.hindawi.com
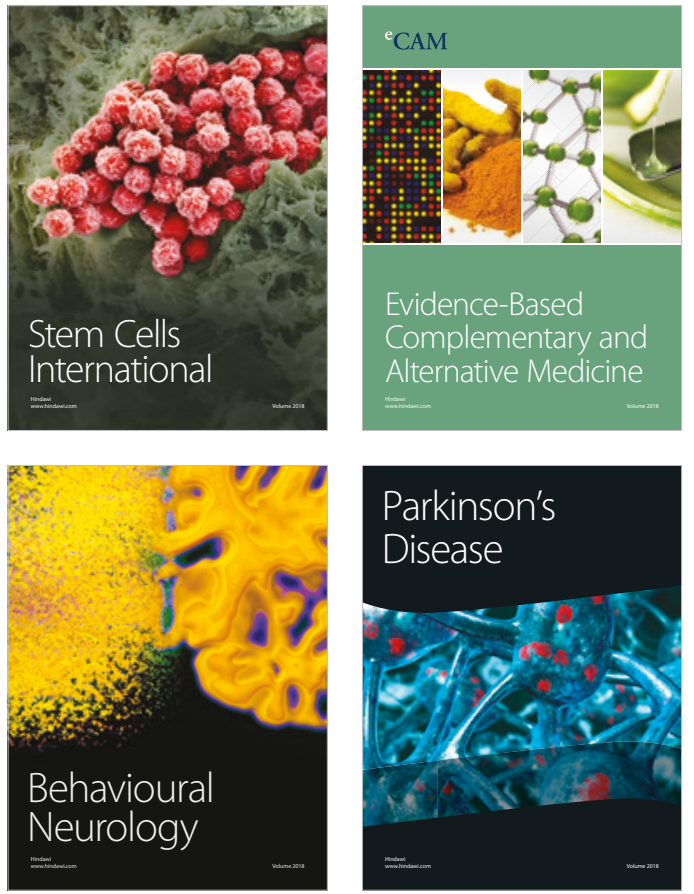

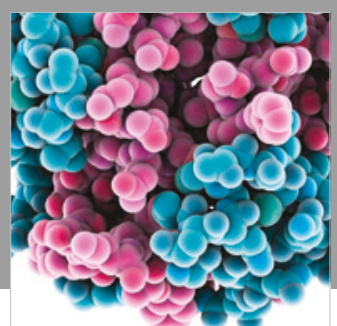

ournal of

Diabetes Research

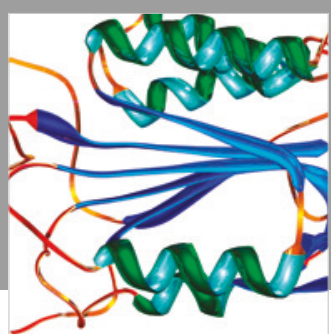

Disease Markers
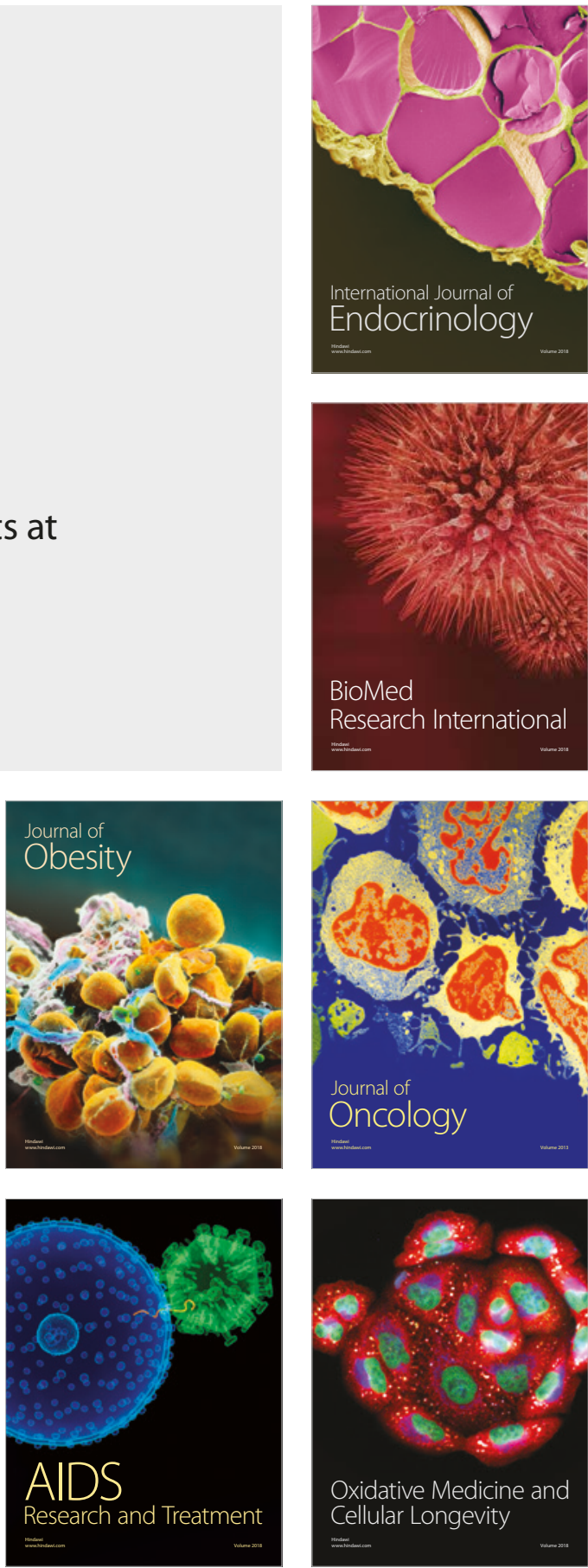